\title{
Natural Variation of Dt2 Promoter Controls Plant Height and Node Number in Semi-Determinant Soybeans
}

\section{Kun Kou}

Northeast Institute of Geography and Agroecology Chinese Academy of Sciences

\section{Tong Su}

Chinese Academy of Sciences

\section{Yanping Wang}

Guangzhou University

Hui Yang

Guangzhou University

Hao Du

Guangzhou University

Milan He

Guangzhou University

Tai Li

Guangzhou University

\section{Lixin Ma}

Guangzhou University

Chunmei Liao

Guangzhou University

\section{Cen Yang}

Guangzhou University

Wenqian Shi

Guangzhou University

\section{Linnan Chen}

Guangzhou University

Yongli Li

Guangzhou university

\section{Bize Yang}

Guangzhou University

\section{Lingping Kong}

Guangzhou University

Shichen Li

Chinese Academy of Sciences

\section{Lingshuang Wang}

Chinese Academy of Sciences

\section{Xiaohui Zhao}

Guangzhou University

Sijia Lu

Guangzhou University

Baohui Liu

Guangzhou University

\section{Fanjiang Kong}

Guangzhou University

Chao Fang ( $\square$ fangchao@gzhu.edu.cn )

Guangzhou University 


\section{Research Article}

Keywords: Soybean, QTL, Genotyping-by-sequencing (GBS), Plant height, Node number, Dt2

Posted Date: March 11th, 2021

DOI: https://doi.org/10.21203/rs.3.rs-278460/v1

License: (c) (7) This work is licensed under a Creative Commons Attribution 4.0 International License. Read Full License 


\section{Abstract}

Soybean [Glycine max (L.) Merrill] is one of the most important legume crops and its plant height (PH) is one significant quantitative trait closely related to node number (NN) and internode length (IL) on the main stem, which affect yield together. In this study, we used a recombinant inbred line (RIL) derived from a cross between two semi-determinate stem soybean varieties (Dt1Dt1Dt2Dt2), JKK378 and HXW. A consistent QTL named $q P H 18$ simultaneously controlling PH, NN, and IL was identified, in which this region harbors the semi-determinant gene, $D t 2$. Sequencing of the promoter of $D t 2$ from JKK378, identified three polymorphisms relative to HXW, including two SNPs and a $18 \mathrm{bp}$ Indel. The expression level of $D t 2$ in $q P H 18^{J K K 378}$ group was lower than that in $q P H 18^{H X W}$ group, meanwhile, as a downstream gene, the expression of $D t 1$ from two groups showed a contrary tendency. Transient transfection assay confirmed that the promoter activity of $D t 2$ from JKK378 is lower compared to HXW. We speculate that the polymorphisms in the two dominant Dt2 promoter caused difference in expression level of $D t 2$ and its downstream gene $D t 1$, hence, affect $\mathrm{PH}, \mathrm{NN}$, IL, and grain weight per plant without changing stem growth habit. Compared to $P H 18^{H X W}$ allele, $q P H 18^{J K K 378}$ allele suppresses the expression of $D t 2$ gene which releases the expression of $D t 1$, thus promotes plant node number and enhanced grain yield. These results will be helpful for revealing the mechanism underlying the node number and plant height, the soybean materials and molecular marker will facilitate molecule breeding.

\section{Introduction}

Soybean [Glycine max (L.) Merrill] is an important economic crop that provides protein and edible oil. Breeding soybean with high yield and quality has always been an important goal pursued by breeders. Soybean yield is a complex quantitative trait, which is affected by plant type consisting of plant height, node number, and internode length and many other traits. It is documented that a significant positive correlation between PH and NN, PH and IL (Liu et al. 2011; Allen et al. 2018; Chang et al. 2018; Assefa et al. 2019). Taller soybean plants produce more NN and IL, which in turn produce more pods. IL and NN affect the strength of the main stem and hence the yield per plant. Plant height and node number on main stem are mainly determined by soybean stem growth habits which is controlled by two major genes, Dt1 and Dt2 (Bernard 1971; Heatherly and Smith 2004). Under the background of Dt1, Dt2 or dt2 genotypes produce semi-determinate and indeterminate phenotypes, respectively. In the $d t 1$ genetic background, the phenotype is determinate, as an epistatic effect of the $d t 1$ allele on the Dt2 locus (Liu et al. 2010; Ping et al. 2014). Studies on near isogenic lines of Dt1 showed that the function of Dt1 gene was regulated by photoperiod, and the phenotype of $D t 1$ gene in regulating stem growth habit was not obvious under short day conditions, but the differences in NN, PH and pod number per plant could be shown under long day conditions (Bernard 1972; Liu et al. 2010). The Dt2 locus has been shown to be encoded by FUL3b gene of the MADS Box transcription factor family, besides, several variation in the first intron was believed to be responsible for the Dt2/ dt2 difference (Ping et al. 2014). It was documented that, highest expression level of Dt2 is in apical stem tips at the V2 stage when the first trifoliate leaflets at node 2 are fully expanded, while the second trifoliate leaflets at node 3 are not yet unfolded (Ping et al. 2014). Furthermore Dt2 directly bind to the Dt1 promoter to inhibit Dt1 transcription, leading to the development and formation of semi-determinate (Liu et al. 2016). Phytochrome E3 and E4 induce the transcription of Dt1 indirectly and thus promote the development of node number and pod number per plant (Xu et al. 2013). Recent research shows that overexpression of FT5a may control plant height and node number development by inducing $A P 1$ transcription and thereby inhibiting the expression of $D t 1$, thus controlling the yield per plant (Takeshima et al. 2019). AP1 inhibit expression of Dt1 to control node number, plant height and pod number per plant development and formation (Chen et al. 2020).

Clarifying the QTLs/genes of node number in soybean is of great significance for increasing node number and yield with appropriate plant height for lodging tolerance like semi-dwarf traits in rice and wheat (Peng et al. 1999; Ashikari et al. 2002; Monna et al. 2002; Hedden 2003). In the past decades, most of the soybean cultivars grown at high latitude are semi-determinate types that may produce more pods per plant than indeterminate cultivars (Setiyono et al. 2007). For a semi-determinate maintains stem elongation after photoperiod induced initiation of floral transition, which allows more overlap between vegetative growth and reproductive development. That provides a better adaptation to a shorter growing season.

Here we used a recombinant inbred line (RIL) population which derived from a cross between two semi-determinate (Dt1Dt1Dt2Dt2) varieties, JKK378 and HXW, to identify QTLs for PH, NN, and IL under LD conditions other than Dt1 and Dt2. Intriguingly, we identified a QTL related to $\mathrm{PH}$ and NN, of which the candidate causal gene is Dt2. qRT-PCR and transient transfection assay confirmed that the polymorphisms on the promoter of $D t 2$ between $q P H 18^{J K K 378}$ and $q P H 18^{H X W}$ group caused the difference in expression level of $D t 2$ and $D t 1$ and the external performance (PH, NN, IL, and grain weight per plant). The results of this study will provide new basic resources and perspectives for further understanding the mechanism of yield of soybean.

\section{Materials And Methods}




\section{Plant materials and field trials}

The JH population of $267 \mathrm{~F}_{6: 8}$ soybean RIL was obtained by single seed descent (SSD) from a cross between JKK378 (e1-

as/e2/E3/E4/Dt1/Dt2) and HXW (e1-as/e2/E3/E4/Dt1/Dt2). The JH population was grown in Harbin ( $\left.45^{\circ} 45^{\prime} \mathrm{N}, 126^{\circ} 380^{\prime} \mathrm{E}\right)$ Heilongjiang province in 2018 and 2019. The population and parents were planted in 2-m-long rows with 15 plants each. The plant height, node number, and internode length were measured and calculated after harvesting. NN and PH was measured from the cotyledonary node to the top of the apical extremity at maturity. And internode length = plant height /node number of mainstem per plant.

\section{Population phenotypic statistical analysis}

Correlation analysis were used to test the significance of the difference in PH, NN, and IL between parents by using SPSS 18.0 (SPSS Inc., Chicago, IL, United States).

\section{DNA isolation}

Fresh and fully developed trifoliate leaves from the parents and the RIL individuals were collected, stored in liquid nitrogen, and then transferred to a $-80^{\circ} \mathrm{C}$ freezer. Total genomic DNA was extracted using a plant genomic DNA kit (CWBIO, Beijing, China). The integrity and quality of the extracted DNA was appraised by $1 \%$ agarose gel electrophoresis. The concentration of DNA in each sample was measured using a Qubit ${ }^{\circledR} 2.0$ fluorometer (Invitrogen, Carlsbad, CA, USA) and a NanoDrop 2000 (Thermo Scientific, Wilmington, DE, USA).

\section{Genotyping by high-throughput sequencing}

For each parent, as previously described, approximately $1.5 \mathrm{ug}$ of DNA was prepared for whole-genome resequencing to generate sequencing libraries (Cheng et al. 2015). Two parental libraries were sequenced on an Illumina Hi-Seq 2500 platform (Illumina, Inc., San Diego, CA, USA), and 150 bp paired end reads with an insert size of approximately 300 bp were developed. Genotypes were identified by low coverage sequencing of the $\mathrm{JH}$ population, which was based on the reference of the parental polymorphic loci. Genomic DNA was incubated at $37^{\circ} \mathrm{C}$ with restriction endonuclease $\mathrm{Msel}$ (New England Biolabs, NEB, Ipswich, MA, USA), T4 DNA ligase (NEB), ATP (NEB), and the Mse/ Yadapter $\mathrm{N}$ containing a barcode. Restriction-ligation reactions were heat-inactivated at $65^{\circ} \mathrm{C}$, and then digested by additional restriction enzymes $\mathrm{Msel}+\mathrm{Nlall}$ at $37^{\circ} \mathrm{C}$. The restriction-ligation samples were purified with Agencourt AMPure (Beckman Coulter, Brea, CA, USA). Polymerase chain reactions (PCR) were conducted using diluted restriction-ligation DNA samples, dNTPs, Phusion Master Mix (NEB) universal primers, and the index primers. The PCR products were purified by Agencourt AMPure XP (Beckman Coulter, Brea, CA, USA) and pooled, then separated by $2 \%$ agarose gel electrophoresis. Fragments ranging in size from 375 to $400 \mathrm{bp}$ (including indicators and adapters) were separated using a gel extraction kit (Qiagen), purified using Agencourt AMPure XP (Beckman, Irvine, CA, United States) and then diluted for sequencing. Then, paired-end sequencing (each end was $150 \mathrm{bp}$ in length) was performed on an Illumina Hi-Seq 2500 system (Illumina, Inc., San Diego, CA, United States) in line with the manufacturer's recommendations.

\section{Sequence data grouping and single nucleotide polymorphisms identification}

Raw data (raw reads) were first processed through a series of quality control (QC) procedures using in-house $\mathrm{C}$ programs to ensure that the reads were reliable without artificial bias. The QC standards included removal of the following: (1) reads with $>50 \%$ bases while Phred quality < 5 ; (2) reads with $\geq 10 \%$ unidentified nucleotides ( $\mathrm{N}$ ); (3) reads that contained Msel and/or Nlalll cut-site remnant sequences; (4) reads with $>10 \mathrm{nt}$ aligned to the adapter, which allowed for $\leq 10 \%$ mismatches; and low-quality raw data were deleted first. The clean reads of each sample were aligned against the reference genome using Burrows-Wheeler Aligner (settings: mem-t4-M-R) (BWA v0.7.10) (Li 2009), then alignment files were converted to BAM by SAMtools software (v1.7.6) (Li et al. 2009). Variant calling of all samples was conducted using GATK (v3.8.0) (Wang et al. 2010). Single nucleotide polymorphism (SNPs) were filtered using a Perl script and were annotated by ANNOVAR (v20170716) based on the GFF files of the reference genome (Wang et al. 2010). Markers with > 30\% missing genotype data, segregation distortion $(P<0.01)$, or abnormal bases were screened out during map construction.

\section{High-density genetic map construction}

Chi-square $\left(\chi^{2}\right)$ tests were carried out to detect segregation distortion for all SNPs. After genotyping, the markers were distributed to 20 linkage groups (or chromosomes) based on their physical position, which was calculated in CM using the QTL IciMapping software (www.isbreeding.net) (Meng et al. 2015).

\section{QTL analysis for PH, NN, and IL using ICIM method}

QTLs for NN, PH and IL were detected using the QTL IciMapping4.2 software with inclusive composite interval mapping (ICIM) to identify QTLs with parameters: PIN (probability in stepwise regression) $=0.001$, step $=1.0 \mathrm{cM}$. Nonsignificant QTLs with logarithm-of-odds (LOD) score values between 2.5 and the permutation test LOD threshold were also included in subsequent analysis and were considered as 
suggestive QTLs (Kong et al. 2018). To eliminate the influence of environment, we calculated the correlation coefficients of NN and PH from the RIL in 2018 and 2019. The results showed that PH had intensive correlation over 2 years, as was NN (Supplementary Table 1). Therefore, we also used the average data of PH and NN to detect QTLs, so as IL.

\section{qRT-PCR analysis of Dt2 and Dt1}

RNA of stem apical meristem (SAM) at V2 stage was extracted using TRIZOL Reagent (Ultrapure RNA Kit, CWBIO, China). The extracted RNA is used to produce reverse transcribe cDNA using the kit (TransScript ${ }^{\circledR}$, China). Then cDNA was performed on the light Cycle 480 system (Roche, Roche Diagnosics, Rotkreuz, Switzerland) using TBGreen $\rrbracket$ qPCR Mix (Vazyme biotech, Nanjing, China). Using Gmtublin as internal reference, the expression level of $D T 2$ and $D t 1$ genes was determined by the following formula (Song et al. 2013): Relative Expression $=2 \Delta C t$, $[\Delta \mathrm{Ct}=\mathrm{Ct}($ Gmtublin) $-\mathrm{Ct}$ (target genes)]. Dt2-specific primers for qRT-PCR were designed with Primer Premier 5.0. Primers was listed in Supplementary Table 11.

\section{Transient transformation vector construction and transient transformation of Arabidopsis protoplasts}

Two different promoter sequences were cloned with specific primers using JKK378 and HXW as template. The purified and recovered promoter sequence fragments were assembled into $p$ Green-0800 vector using Exnase $₫ \bigotimes$ (ClonExpress $₫ \square$ One Step Cloning Kit, Vazyme, Nanjing, China). The vector included Renilla luciferase (REM) initiated by $35 S$ and luciferase $(L U C)$ started by the target promoter. The recombined vector transformed into $E$. coli DH5a. Correct sequencing of positive plasmids transformed Arabidopsis protoplasts with PEGmediated method (Lu et al. 2020). In this study, the active and extended leaves of Columbia wild-type Arabidopsis were selected for the preparation of protoplasm. Fluorescence detection was performed 18 hours after conversion using microplate reader (Biotek Synergy H1, USA). Promoter activity was measured by the ratio of LUC divided by REN.

\section{Results}

\section{Statistics of phenotypes}

The parents had the same genotype (e1-as/e2/E3/E4/Dt1/Dt2) in the major flowering time genes of E1-E4 and the stem growth habit genes of Dt1 and Dt2. Their PH and NN were significant differences both in 2018 and 2019 (Table 1 and Fig. 1) and the RIL showed transgressive separation (Supplementary Fig. 1). Although there is no difference between parents in IL trait, while RIL showed transgressive separation (Table 1 and Supplementary Fig. 1). The results showed that difference of plant height among parents was mainly caused by nodes. The absolute value of skewness of the mean value of the two traits in the two years was $<1$, and the three phenotypes of the RIL presented approximately normal distribution (Table 1), which indicating that these traits are quantitatively controlled by multiple loci. The data of $\mathrm{PH}$ and NN had intensive correlation over 2 years, as was IL (Supplementary Table 1). Therefore, we also used the average data of PH, NN, and IL to detect QTLs. 
Table 1

Statistical analysis result of the parents and the whole JH population from 2018 to 2019

\begin{tabular}{|c|c|c|c|c|c|c|c|c|c|c|c|c|}
\hline \multirow[t]{2}{*}{ Trait } & \multirow[t]{2}{*}{ Environment } & \multicolumn{4}{|l|}{ Parents } & \multicolumn{7}{|l|}{ RIL } \\
\hline & & JKK378 & HXW & Range & $\begin{array}{l}P \\
\text { value }\end{array}$ & Mine & $\operatorname{Max}^{f}$ & Range $^{\mathrm{g}}$ & Mean ${ }^{\mathrm{h}}$ & CV\% ${ }^{i}$ & Skewness & Kurtosis \\
\hline \multirow[t]{3}{*}{$\mathrm{PH}^{\mathrm{a}}$} & 2018 & 115.1 & 76 & 39.1 & $\begin{array}{l}2.57 \mathrm{E}- \\
04\end{array}$ & 82.5 & 198.8 & 116.3 & 139.43 & $19 \%$ & 0.41 & -0.52 \\
\hline & 2019 & 112.55 & 66.3 & 46.25 & $\begin{array}{l}3.79 \mathrm{E}- \\
09\end{array}$ & 62.2 & 178.7 & 116.47 & 123.02 & $31 \%$ & -0.01 & -0.03 \\
\hline & Average $\mathrm{PH}^{\mathrm{b}}$ & 112.5 & 71.5 & 41 & $\begin{array}{l}2.07 E- \\
07\end{array}$ & 73.7 & 186.2 & 112.51 & 132.08 & $17 \%$ & 0.28 & -0.42 \\
\hline \multirow[t]{3}{*}{ NN } & 2018 & 24 & 16.4 & 7.6 & $\begin{array}{l}2.67 \mathrm{E}- \\
04\end{array}$ & 17.33 & 27 & 9.67 & 22.05 & $12 \%$ & 0.04 & 0.08 \\
\hline & 2019 & 23.75 & 14.2 & 9.55 & $\begin{array}{l}1.11 \mathrm{E}- \\
08\end{array}$ & 15.8 & 29 & 13.2 & 20.94 & $28 \%$ & 0.50 & 0.94 \\
\hline & Average $\mathrm{NN}^{\mathrm{C}}$ & 23.87 & 15.3 & 8.57 & $\begin{array}{l}5.56 \mathrm{E}- \\
07\end{array}$ & 17.53 & 26.3 & 8.77 & 21.54 & $9 \%$ & 0.17 & 0.26 \\
\hline \multirow[t]{3}{*}{ IL } & 2018 & 4.82 & 4.66 & 0.16 & $\begin{array}{l}6.80 \mathrm{E}- \\
01\end{array}$ & 4.02 & 9.97 & 5.95 & 6.35 & $20 \%$ & 0.51 & -0.24 \\
\hline & 2019 & 4.63 & 4.67 & -0.04 & $\begin{array}{l}6.90 E- \\
01\end{array}$ & 3.49 & 8.38 & 4.89 & 5.88 & $30 \%$ & 0.42 & -0.04 \\
\hline & Average $\mathrm{IL}^{\mathrm{d}}$ & 4.72 & 4.66 & 0.06 & $\begin{array}{l}7.20 \mathrm{E}- \\
01\end{array}$ & 3.76 & 8.89 & 5.13 & 6.14 & $16 \%$ & 0.55 & 0.08 \\
\hline
\end{tabular}

a Plant height which was measured as the distance of main stem in $\mathrm{cm}$ from the cotyledonary node to the top of the apical extremity at maturity.

${ }^{\mathrm{b}}$ The average data of plant height in two environments (2018 and 2019 in Harbin).

CThe average data of node number in two environments (2018 and 2019 in Harbin).

dThe average data of internode length in two environments (2018 and 2019 in Harbin).

eMinimum value of the population.

${ }^{f}$ Maximum value of the population.

gThe difference between the minimum and maximum values.

${ }^{\mathrm{h}}$ The average data of the whole population.

${ }^{i}$ Coefficient of variance in percentage type.

Analysis of sequencing data and genetic linkage maps construction

JKK378 and HXW were sequenced at an average depth of $15.61 \times$ and $13.42 \times$, and generated 14,817,826,500 and 12,740,326,800 bases, respectively. We found $92.93 \%$ and $92.85 \%$ of the total reads were of high quality, with an average Q30 ratio and GC content of $35 \%$ and $36 \%$, respectively. The coverage of each parental cultivar reads mapped to the reference genome were both $91.60 \%$ (Supplementary Table 2 ). We obtained a total of 647,829 SNPs. Ultimately, a total of 4031 SNP markers was used to construct a linkage map covering $2909.01 \mathrm{cM}$, with an average marker interval of $0.72 \mathrm{~cm}$ (Supplementary Fig. 2 and Supplementary Table 3).

\section{QTL mapping of plant height, node number, and internode length}

The threshold of the LOD score of ICIM method to evaluate the statistical significance of the QTL effect was shown in Supplementary Table 4. Common loci located in adjacent regions and have same effect in two years were considered as a consistent QTL.

In this study, a QTL associated with PH trait, namely, qPH18 (QTL for plant height on chromosome 18) was detected over two years (2018 and 2019), and as expected the QTL was detected in average data of two years. It spanned a physical region of 53,950,157 bp-56,334,200 bp to the reference genome, accounted for $21.69 \%$ of the observed phenotypic variation (PVE) and increased 10.87 centimeter (CM) with 
max LOD score of 16.95 in year 2018 (Table 2). In year 2019, it was detected at a physical region of 54,577,297 bp-55,740,447 bp on chromosome 18 with max LOD score of 8.92 and explained $12.56 \%$ of the observed PVE. The QTL named qNN18 for NN traits were detected over two years (2018 and 2019) and in average data of two years. It explained $7.16 \%, 10.90 \%$, and $8.17 \%$ of the observed PVE with max LOD score of $5.68,9.92$, and 8.99 and their additive effect were $0.51,0.67$, and 0.50 , respectively. QTL qIL 18 for IL trait, explained $2.90 \%, 11.30 \%$, and $13.12 \%$ of the observed PVE with additive effect of $0.37,0.34$, and 0.33 , and its Max LOD were 9.05, 9.46, and 9.47, respectively. QTL physical regions of the IL trait on chromosome 18 overlapped with those of the NN and PH traits. We concluded that the QTLs named qPH18, qNN18, and qIL 18 which were detected on chromosome 18, were the same QTL simultaneously regulating PH, NN, and IL and were the major QTL in the JH RIL population (Table 2 and Supplementary Fig. 3). The allele of qPH18 was from JKK378, resulting in more node, longer plant height and internode length. Another QTL for plant height was detected at a similar position as the QTL for IL on chromosome 4, named qPH4.2. qPH4.2 was detected in Harbin in 2018 and 2019, as well as the average data of the two growth seasons (Supplementary Table 5 and Supplementary Fig. 3). qPH4.2 was located between markers 4-MAR-21 to 4-MAR-22, at physical position of 2,452,312 bp$2,443,173 \mathrm{bp}$. It explained $4.54 \%, 4.93 \%$, and $2.56 \%$ of the observed PVE with max LOD score of $4.16,4.08$, and 3.28 and their additive effect were 4.95, 4.12, and 3.65, respectively. A minor $q P H 9$ was located between markers 9-MAR-27 to 9-MAR-28 on chromosome 9, at a similar physical position of 4,970,193-6,972,683 as the QTL for NN. It explained $2.8 \%$ of the observed PVE with max LOD score of 2.5 and the additive effect was 3.91(Supplementary Table 5 and Supplementary Fig. 3).

Table 2

Detail information about the major QTL for PH, NN, and IL detected by ICIM in JH population on chromosome 18

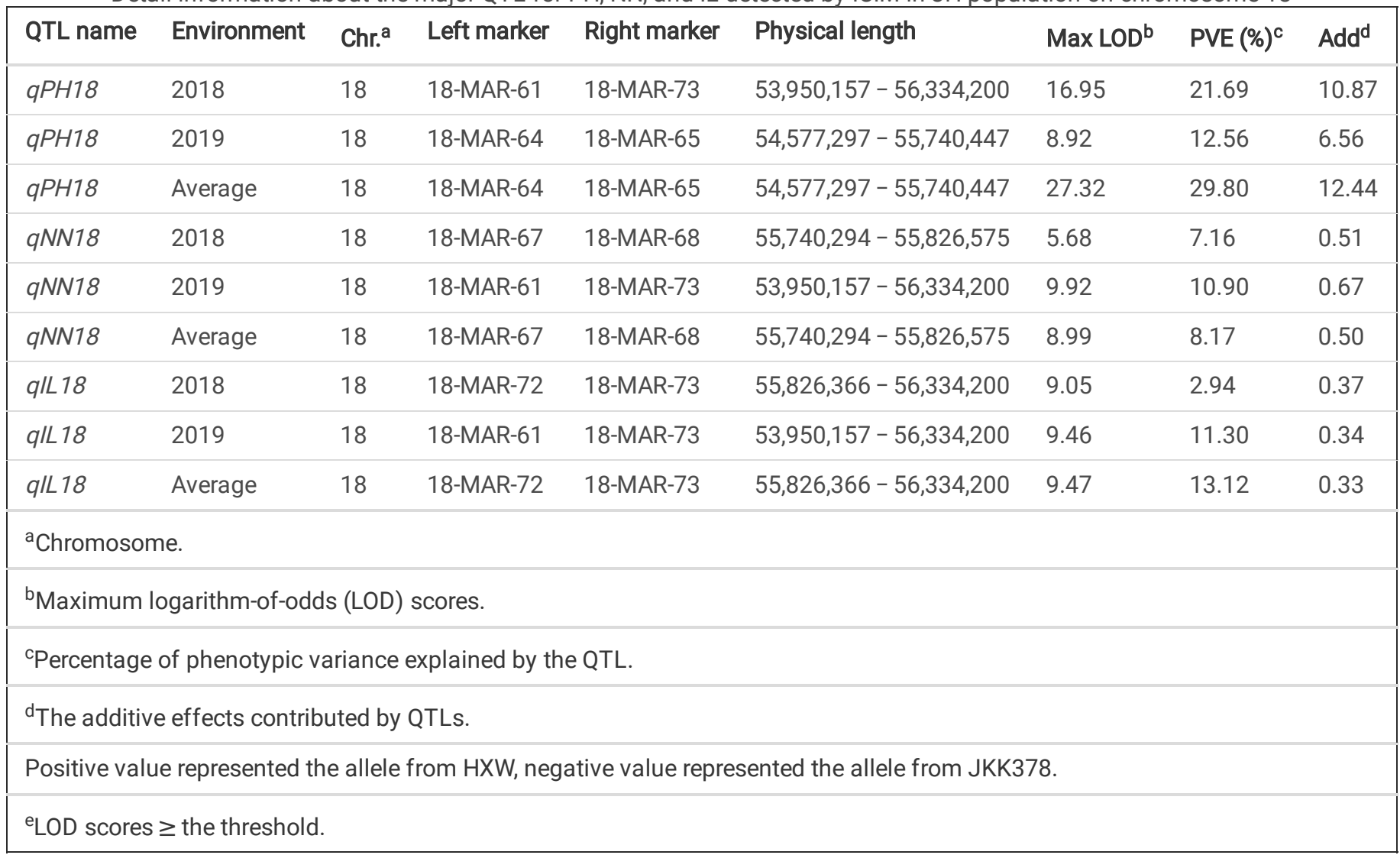

\section{Candidate genes prediction}

To summarize, we detected one major QTL on the end of the short arm of chromosome 18 related to PH, NN and IL. We combined the intervals of these interrelated QTLs to obtain a larger QTL interval for mining candidate genes. To identify candidate genes, we adopted a method of combining the genes in the larger QTL regions with interparental gene variation, Gene Ontology (GO) annotation, and functional annotation of orthologues in Arabidopsis.

A total of 295 genes were screened in the major $q P H 18$ intervals 53,950,157bp-56,334,200bp on chromosome 18 according to the Gmax_275_Wm82.a2.v1 (Schmutz et al. 2010). According to SNPs and Indel calling of the results of the resequencing of the parents, 247 SNPs resulted in amino acid changes or frameshift mutations were in exons of 54 genes (Supplementary Table 6). Then, among 295 genes, 255 genes could be functionally annotated using WEGO2.0 (Supplementary Table 7). Of these, 161, 62, and 223 genes were functionally annotated to the categories of biological processes, cellular components, and molecular function, respectively (Supplementary Fig. 4). Of these genes, ten genes were related to transcription factor activity including Dt2 (Glyma.18G273600), were considered major candidate 
genes. In addition, compared 295 genes, two of these were associated with plant growth and development based on the annotation information and had mutations in the coding sequence (CDs) region (Supplementary Table 8). Glyma.18G275200 encodes auxin transport protein, which was involved in the transport of auxin. The homologous gene in Arabidopsis is AT3G02260, whose homologous mutants are characterized by organ elongation defects (dwarfism), altered root structure, decreased apical dominance, defective light response, and abnormal auxin transport (Kasajima et al. 2006; Nobutoshi et al. 2007; Ivanova et al. 2014). Glyma.18G282000 is related to ARF GTPaseactivating protein, and the homologous gene in rice is Os02g0198300 which interferes with auxin influx, vesicle trafficking and root development (Zhuang et al. 2010). These genes above were classified as the most likely candidate genes (Supplementary Table 8).

\section{Expression analysis of Dt2 of qPH18 for PH, NN, and IL}

According to GBS sequencing results, RIL were divided into two subgroups which possessing homozygous alleles of JKK378 and HXW at $q P H 18$, respectively. Significant differences were detected by analysis of variance for plant height, node number, internode length, and grain weight per plant in year of 2018 and 2019, which showed that subgroup harboring the allele of $q P H 18$ JKK378 increased PH, NN, and IL, and ultimately leaded to an increase in yield (Fig. 2). The list of candidate genes of $q P H 18$ included Dt2, despite two parents are both semideterminate sharing $D t 2$, we still counted it as a probable candidate gene due to its key role in regulating node number, plant height and growth habits (Ping et al. 2014).Thus, we sequenced and analyzed genomic sequences of $3 \mathrm{kp}$ promoter sequence between JKK378 and HXW. Three polymorphisms were detected in Dt2 promotor, including two SNPs and one Indel. The polymorphisms of SNP_-2570 (T-A), SNP_-2420 (T-C), and Indel_-2376 (TATATATATATATATATA) leaded to changes in a cis-acting elements POLLEN1LELAT52 -motif (sense strand), a CAAT-box (sense strand), and a TATA-box (sense strand), according to NEW PLACE analysis. These variations in the promoter of two dominant Dt2 allele from JKK378 and HXW might lead to a difference in the level of expression.

To test this possibility, we first examined the expression level of $D t 2$ in stem apical meristem (SAM) at V2 stage from two parents and two subgroups divided by the polymorphisms of the promoter of $D t 2$. The results showed that expression level of Dt2 from JK378 were significantly lower than that of HXW, meanwhile, several random samples of subgroup harboring the allele of $q P H 18^{J K K 378}$ had significantly lower relative expression of Dt2 (Fig. 3a). In addition, we detected the expression level of $D t 1$ between these two subgroups. As expected, the expression level of $D t 1$ in subgroup harboring the allele of $q P H 18^{H X W}$ was inhibited (Fig. 3b). These followed the model of $D t 2$ inhibit $D t 1$ and then regulate node number and plant height.

To validate the functional difference of Dt2 promoters, we performed a transient transfection assay to confirm whether polymorphisms on the $D t 2$ promoter caused a difference in promoter activity. The two different promoter sequences were cloned and introduced into vector pGreen0800-LUC to generate reporter constructs (Fig. 3c). Subsequently, plasmids containing the reporter were transformed into Arabidopsis protoplasts. After 18 hours, the activities of LUC and REN of two reporter constructs were detected by a microplate assay, respectively. Twoway ANOVA result ( $P=2.67 \mathrm{E}-03)$ of the two ratios (LUC/REN) was significantly different. The activity of JKK378 promoter was significantly lower than that of HXW promoter (Fig. 3d), which suggest that sequence variations in promoters leaded to Dt2 functional intensity difference. Taken together, we concluded that $D t 2$ was the candidate gene of $q P H 18$, while a fine mapping is still need.

In addition, we developed a dCAPs molecular marker focused on SNP_-2420 (T-C) (Supplementary Fig. 5). PCR products obtained by two rounds of specific primers and that of HXW was digested by restriction enzyme Mlu After digested, the HXW genotype fragment was 172bp while the JKK378 genotype fragment was 197bp. Result confirmed that the dCAPs marker could be used for the genotyping of two different promoters between JKK378 and HXW, which have a broad application prospect in molecular breeding research.

\section{Discussion}

A genetic map constructed by RIL plays a crucial role in identifying QTLs of the interesting traits. With the emergence of new molecular markers, high-throughput sequencing has become the optimization solution for obtaining SNP markers and constructing high-density linkage maps for QTL mapping (Zhou et al. 2016; Kong et al. 2018; Fang et al. 2019). In the present study, we constructed a high-density genetic map could be helpful for QTL analysis. Based on this map, we detected several QTLs associated with PH, NN, and IL, and identify candidate genes with multiple methods.

$\mathrm{PH}$ is an important yield-related agronomic trait in soybean. It is documented that $\mathrm{PH}$ and $\mathrm{NN}$ as well as $\mathrm{PH}$ and IL were positively correlated (Liu et al. 2011; Allen et al. 2018; Chang et al. 2018; Assefa et al. 2019). Therefore, due to the pleiotropic effect, the same QTL may affect multiple traits. In the present study, we identified a major QTL that affect PH, NN, and IL, simultaneously, named $q P H 18$ which located on the terminal of chromosome 18 with physical region of 53,950,157 bp-56,334,200 bp (Table 2 and Supplementary Fig. 3). This interval was identified multiple times and is also associated with some other traits, such as pod maturity, lodging, seed yield, and seed oil (Supplementary 
Table 9) (Kabelka et al. 2004; Sun et al. 2006; Guzman et al. 2007; Qi et al. 2011; Kim et al. 2012). However, most QTLs only work on PH and NN or PH and IL, such as, $q P H 9$ is related to PH and NN, and $q P H 4.2$ affected PH and IL (Supplementary Fig. 3 and Supplementary Table 5). These results showed that plant height was not affected by either node number or internode length alone, but by the joint effect of both, which makes the genetic dissection of plant height trait more complicated. In addition, an environmentally specific $q P H 9$ was detected only in 2018, we examined some meteorological data such as effective accumulated temperature, day length, and rainfall over the developmental stage in 2018 and 2019, and we found that there were significantly different in June and July (flowering period) in two years about effective accumulated temperature and rainfall (Supplementary Table 10). That might be helpful to account for QTL were only detected within a year.

A series of QTLs/loci associated with PH have already been reported. Up to now, two important genes, Dt1 and Dt2, affect PH by regulating stem growth habit (Liu et al. 2010; Ping et al. 2014). Dt2 could directly interact with the Dt1 promoter to repress Dt1 transcription, resulting in a semi-determinate growth habit affecting $\mathrm{PH}$. In our research, we mapped a major QTL qPH18 on chromosome 18 and Dt2 is within this region as well as some known Arabidopsis plant height homologous genes and transcription factor (Supplementary Table 8 ). The phenotypic values of $\mathrm{PH}, \mathrm{NN}, \mathrm{IL}$, and grain weight per plant presented obvious differences in two subgroups harboring the allele from parents of $q P H 18$, suggesting that $q P H 18$ eventually increased yield by affecting PH, NN, and IL (Fig. 2). And this QTL deserves further study and can be used in subsequent breeding. Despite, two parents both have semi-determinate stem growth habit which indicated that they shared dominant Dt2, we still analyzed the expression level of Dt2 at V2 stage in two subgroups on account of Dt2 is an important regulator of node number, plant height and even growth habits, and the expression level of Dt2 showed a corresponding difference (Fig. 3a). Whereafter transient transfection assay also showed that the promoter activity of JKK378 and HXW were different, which was consistent with gene expression level (Fig. 3d). These results together suggested that Dt2 was the candidate gene of the $q P H 18$, and even that the known and already applied dominant $D t 2$, is not a single functional strength allele, there are at least two different-intensity dominant $D t 2$ allele in nature. Classical genetic analyses showed that the functional strength (Dt1 or $d t 1$ ) and expression level of $D t 1$ regulated by $D t 2$, determine soybean node number, plant height, and stem growth habit. Here we speculated that there exist a threshold of the functional strength and expression level of DT1 which corresponds to semi-determinate growth habit (Dt1Dt1Dt2Dt2). Between this threshold and $d t 1 d t 1$ (determinate growth habit), there exist various expression level of Dt1 due to various expression level of Dt2, which finally appear as different node number and plant height. This provided a new sight of molecular breeding focus on node number and plant height by using dominant $D t 2$ with different promoter activity.

Fine mapping of the above QTLs will be helpful for elucidating the molecular mechanisms of the underlying regulating PH, NN and IL. Our study will provide fundamental resources for revealing the mechanism underlying the node number and plant height, the soybean materials and molecular marker will facilitate molecule breeding.

\section{Declarations}

Ethics approval and consent to participate Not applicable.

Consent for publication Not applicable.

Data and material availability Not applicable.

Conflict of interest statement Authors declare no competing interests.

Funding information This work was supported by National Natural Science Foundation of China (31930083) and EUCLEG Horizon 2020 of European Union (No.727312). This work was also funded by the National Key Research and Development Program (Nos. 2017YFE0111000), National Natural Science Foundation of China $(31701445,31930083,31725021,31801384)$ and the Major Program of Guangdong Basic and Applied Research (2019B030302006), Open Project Foundation of National Key Laboratory for Crop Genetics and Germplasm Enhancement (ZKW201901).

Author contributions $\mathrm{CF}$, FK, and BL designed the experiments. KK, TS, YW, HY, HD, MH, TL, LM, CL, CY, WS, LC, YL, BY carried out the experiments. KK, LK, SL, LW, XZ, SL, BL, FK, and CF analyzed the data. KK, FK, BL, and CF wrote the paper.

Acknowledgements We would like to acknowledge Mrs. Yafeng Liu for phenotyping and managing the field.

\section{Author information}

Kun Kou, Tong Su1, Yanping Wang contributed equally to this work.

\section{Affiliations}


The Innovative Academy of Seed Design, Key Laboratory of Soybean Molecular Design Breeding, Northeast Institute of Geography and Agroecology, Chinese Academy of Sciences, Harbin, China

Kun Kou, Tong Su, Shichen Li, Lingshuang Wang, Baohui Liu, Fanjiang Kong

Innovative Center of Molecular Genetics and Evolution, School of Life Sciences, Guangzhou University, Guangzhou, China

Hui Yang, Hao Du, Milan He, Tai Li, Lixin Ma, Chunmei Liao, Cen Yang, Wenqian Shi, Linnan Chen, Yongli Li, Bize Yang, Lingping Kong, Xiaohui Zhao, Sijia Lu, Baohui Liu, Fanjiang Kong, Chao Fang

\section{University of Chinese Academy of Sciences, Beijing, China}

Kun Kou, Tong Su, Shichen Li, Lingshuang Wang

\section{Mudanjiang Branch of Heilongjiang Academy of Agricultural Sciences, Mudanjiang, China}

Yanping Wang

\section{Corresponding authors}

Correspondence to Chao Fang or Fanjiang Kong or Baohui Liu

\section{References}

1. Allen LH, Zhang L, Boote KJ, Hauser BA (2018) Elevated temperature intensity, timing, and duration of exposure affect soybean internode elongation, mainstem node number, and pod number per plant. The Crop Journal 6:148-161. https://doi.org/https://doi.org/10.1016/j.cj.2017.10.005

2. Ashikari M, Sasaki A, Ueguchi-Tanaka M, et al (2002) Loss-of-function of a Rice Gibberellin Biosynthetic Gene, GA20 oxidase (GA20ox2), Led to the Rice “Green Revolution." Breeding Science 52:143-150

3. Assefa T, Otyama PI, Brown A V, et al (2019) Genome-wide associations and epistatic interactions for internode number, plant height, seed weight and seed yield in soybean. BMC Genomics 20: 527

4. Bernard RL (1971) Two Major Genes for Time of Flowering and Maturity in Soybeans1. Crop Science - CROP SCI 11: 242-244. https://doi.org/10.2135/cropsci1971.0011183X001100020022x

5. Bernard RL (1972) Two Genes Affecting Stem Termination in Soybeans1. Crop Science 12: 235-239

6. Chang F, Guo C, Sun F, et al (2018) Genome-Wide Association Studies for Dynamic Plant Height and Number of Nodes on the Main Stem in Summer Sowing Soybeans. Frontiers in plant science 9:1184. https://doi.org/10.3389/fpls.2018.01184

7. Chen L, Nan H, Kong L, et al (2020) Soybean AP1 homologs control flowering time and plant height. Journal of Integrative Plant Biology 62(12): 1868-1879

8. Cheng W, Liu F, Li M, et al (2015) Variation detection based on next-generation sequencing of type Chinese 1 strains of Toxoplasma gondii with different virulence from China. BMC genomics 16:888. https://doi.org/10.1186/s12864-015-2106-z

9. Fang C, L C, H N (2019) Rapid identification of consistent novel QTLs underlying long-juvenile trait in soybean by multiple genetic populations and genotyping-by-sequencing. Molecular breeding 39: 80

10. Guzman PS, Diers BW, Neece DJ, et al (2007) QTL Associated with Yield in Three Backcross-Derived Populations of Soybean. Crop Science 47:111. https://doi.org/10.2135/cropsci2006.01.0003

11. Heatherly L, Smith J (2004) Effect of soybean stem growth habit on height and node number after beginning bloom in the midsouthern USA. Crop Science 44:1855-1858. https://doi.org/10.2135/cropsci2004.1855

12. Hedden P (2003) The genes of the Green Revolution. Trends in Genetics 19:5-9

13. Ivanova A, Law SR, Narsai R, et al (2014) A Functional Antagonistic Relationship between Auxin and Mitochondrial Retrograde Signaling Regulates Alternative Oxidase1a Expression in Arabidopsis. Plant Physiology 165:1233

14. Kabelka EA, Diers BW, Fehr WR, et al (2004) Putative alleles for increased yield from soybean plant introductions. Crop Science 784791. https://doi.org/10.2135/cropsci2004.0784

15. Kasajima, Naoko, Ohkama-Ohtsu, et al (2006) The BIG gene is involved in regulation of sulfur deficiency-responsive genes in Arabidopsis thaliana. Physiologia Plantarum 129 (2): 351-363 
16. Kim KS, Diers BW, Hyten DL, et al (2012) Identification of positive yield QTL alleles from exotic soybean germplasm in two backcross populations. Theoretical \& Applied Genetics 125:1353-1369. https://doi.org/10.1007/s00122-012-1944-1

17. Kong L, Lu S, Wang Y, et al (2018a) Quantitative trait locus mapping of flowering time and maturity in soybean using next-generation sequencing-based analysis. Frontiers in Plant Science 9:1-20. https://doi.org/10.3389/fpls.2018.00995

18. Kong L, Sijia L, Yanping W, et al (2018b) Quantitative Trait Locus Mapping of Flowering Time and Maturity in Soybean Using NextGeneration Sequencing-Based Analysis. Frontiers in Plant Science 9:995-

19. Li H (2009) Fast and Accurate Short Read Alignment with Burrows-Wheeler Transform. Bioinformatics (Oxford, England) 25:1754-1760. https://doi.org/10.1093/bioinformatics/btp324

20. Li H, Handsaker B, Wysoker A, et al (2009) The Sequence Alignment/Map format and SAMtools. Bioinformatics 25:2078-2079. https://doi.org/10.1093/bioinformatics/btp352

21. Liu B, Watanabe S, Uchiyama T, et al (2010) The soybean stem growth habit gene Dt1 is an ortholog of arabidopsis TERMINAL FLOWER1. Plant physiology 153:198-210. https://doi.org/10.1104/pp.109.150607

22. Liu W, Kim MY, Van K, et al (2011) QTL identification of yield-related traits and their association with flowering and maturity in soybean. Journal of Crop Science \& Biotechnology 14:65-70

23. Liu Y, Zhang D, Ping J, et al (2016) Innovation of a Regulatory Mechanism Modulating Semi-determinate Stem Growth through Artificial Selection in Soybean. PLoS Genetics 12(1): e1005818. https://doi.org/10.1371/journal.pgen.1005818

24. Lu S, Dong L, Fang C, et al (2020) Stepwise selection on homeologous PRR genes controlling flowering and maturity during soybean domestication. Nature Genetics 52:1-9. https://doi.org/10.1038/s41588-020-0604-7

25. Meng L, Huihui, Li, et al (2015) QTL IciMapping:Integrated software for genetic linkage map construction and quantitative trait locus mapping in biparental populations. The Crop Journal 269-283. https://doi.org/CNKI:SUN:CROP.0.2015-03-011

26. Monna L, Noriyuki K, Rika Y, et al (2002) Positional Cloning of Rice Semidwarfing Gene, sd-1: Rice "Green Revolution Gene" Encodes a Mutant Enzyme Involved in Gibberellin Synthesis. Dna Research 1 9(1):11-17

27. Nobutoshi Y, Mitsuhiro S, Hidehiro F, et al (2007) CRM1/BIG-Mediated Auxin Action Regulates Arabidopsis Inflorescence Development. Plant \& Cell Physiology 1275-1290

28. Peng J, Richards DE, Hartley NM, et al (1999) “Green revolution” genes encode mutant gibberellin response modulators. Nature 400:256-261

29. Ping J, Liu Y, Sun L, et al (2014a) Dt2 Is a Gain-of-Function MADS-Domain Factor Gene That Specifies Semideterminacy in Soybean. Plant Cell 26:2831-2842

30. Ping J, Liu Y, Sun L, et al (2014b) Dt2 is a gain-of-function MADS-domain factor gene that specifies semideterminacy in soybean. Plant Cell 26:2831-2842. https://doi.org/10.1105/tpc.114.126938

31. Qi ZM, Wu Q, Han X, et al (2011) Soybean oil content QTL mapping and integrating with meta-analysis method for mining genes. Euphytica 179:499-514. https://doi.org/10.1007/s10681-011-0386-1

32. Schmutz J, Cannon SB, Schlueter J, et al (2010) Genome sequence of the palaeopolyploid soybean. Nature 463:178-183. https://doi.org/10.1038/nature08670

33. Setiyono T, Weiss A, Specht J, et al (2007) Understanding and modeling the effect of temperature and daylength on soybean phenology under high-yield conditions. Field Crops Research 100:257-271. https://doi.org/10.1016/j.fcr.2006.07.011

34. Song, QX, QT, et al (2013) Soybean GmbZIP123 gene enhances lipid content in the seeds of transgenic Arabidopsis plants. J EXP BOT 64(14):4329-4341

35. Sun D, Li W, Zhang Z, et al (2006) Quantitative trait loci analysis for the developmental behavior of Soybean (Glycine max L. Merr.). TAG Theoretical and applied genetics Theoretische und angewandte Genetik 112:665-673. https://doi.org/10.1007/s00122-005-0169-y

36. Takeshima R; Nan H; Harigai K; Dong L (2019) Functional divergence between soybean FLOWERING LOCUS T orthologues, FT2a and FT5a, in post-flowering stem growth. Journal of experimental botany 67:5247-5258

37. Wang K, Li M, Hakonarson H (2010) ANNOVAR: functional annotation of genetic variants from high-throughput sequencing data. Nucleic Acids Research 38:164-164. https://doi.org/10.1093/nar/gkq603

38. Xu M, Xu Z, Liu B, et al (2013) Genetic variation in four maturity genes affects photoperiod insensitivity and PHYA-regulated postflowering responses of soybean. Bmc Plant Biology 13:91

39. Zhou Z, Zhang C, Zhou Y, et al (2016) Genetic dissection of maize plant architecture with an ultra-high density bin map based on recombinant inbred lines. BMC Genomics 17:1-15. https://doi.org/10.1186/s12864-016-2555-z 
40. Zhuang X, Jiang J, Li J, et al (2010) Over-expression of OsAGAP, an ARF-GAP, interferes with auxin influx, vesicle trafficking and root development. Plant Journal 48:581-591

\section{Figures}

a
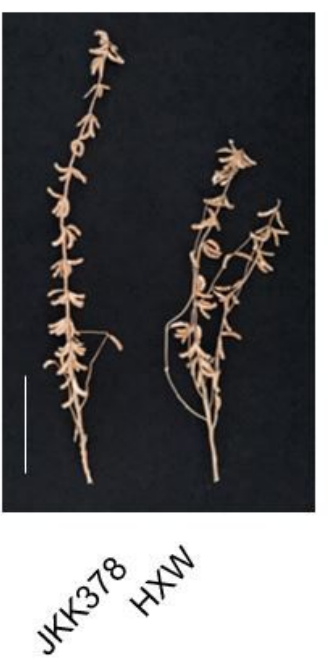

b
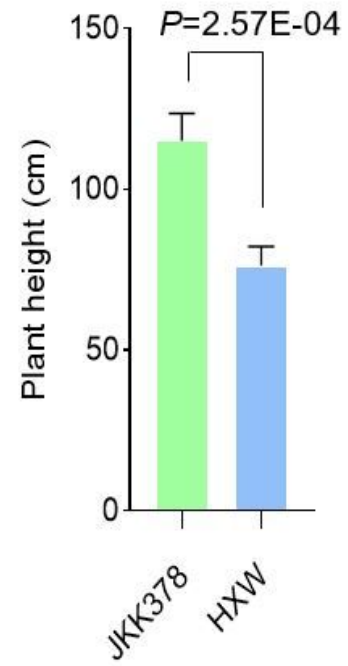

e

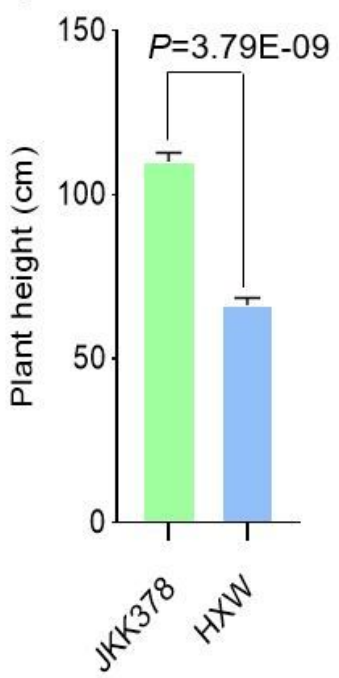

C
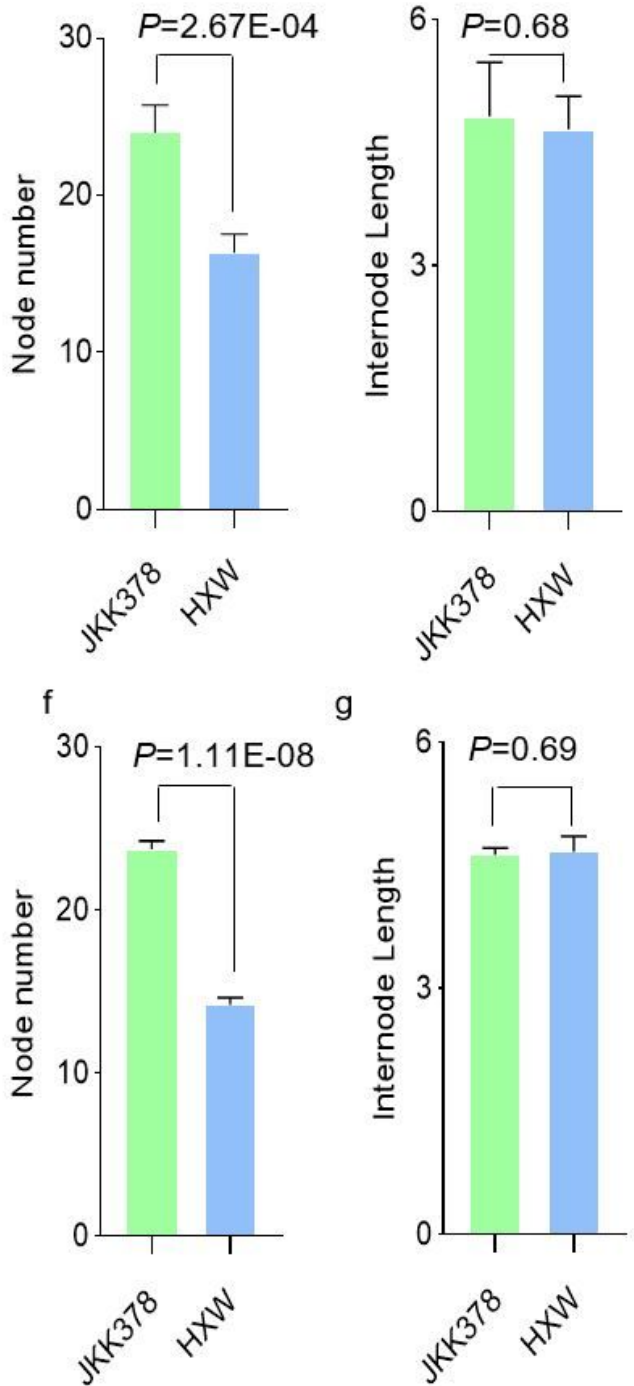

\section{Figure 1}

Phenotype of the parents of JH population. Left is JKK378 and right is HXW. a: Plants of JKK378 and HXW fully matured. b, e; Plant height data of the parents in 2018 and 2019 in Harbin. c, f: Node number data of the parents in 2018 and 2019 in Harbin. d, g: Internode length data of the parents in 2019 in Harbin. Bar=20cm. 
a

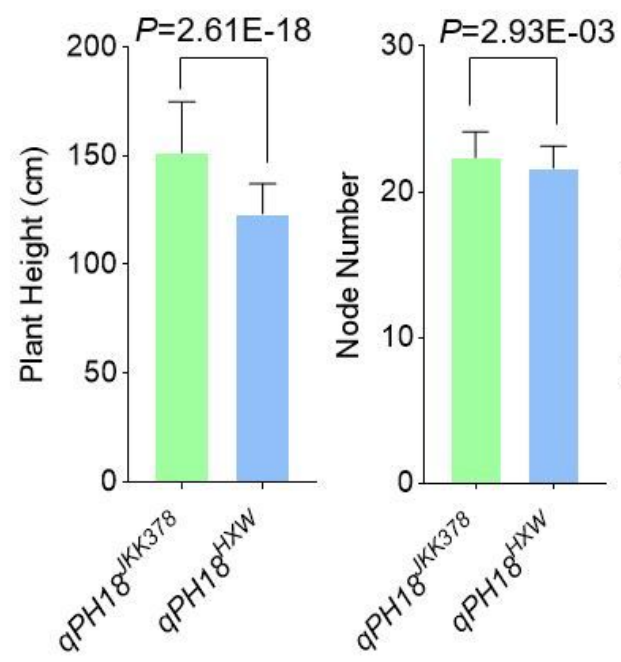

b
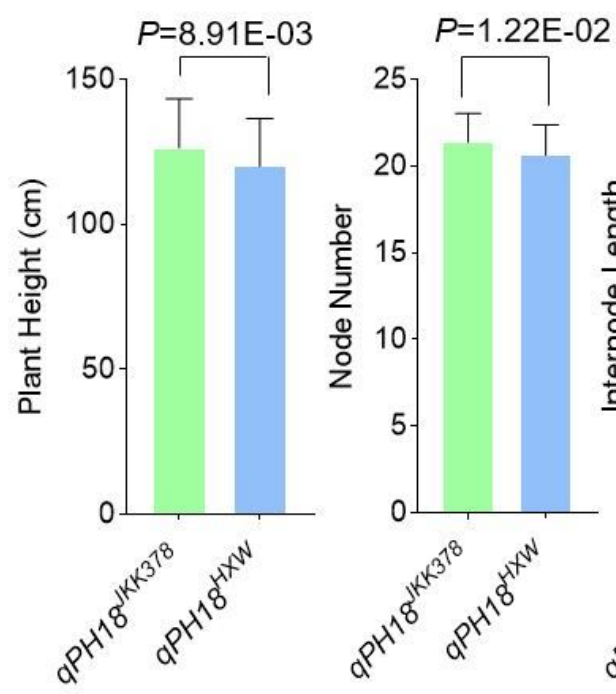
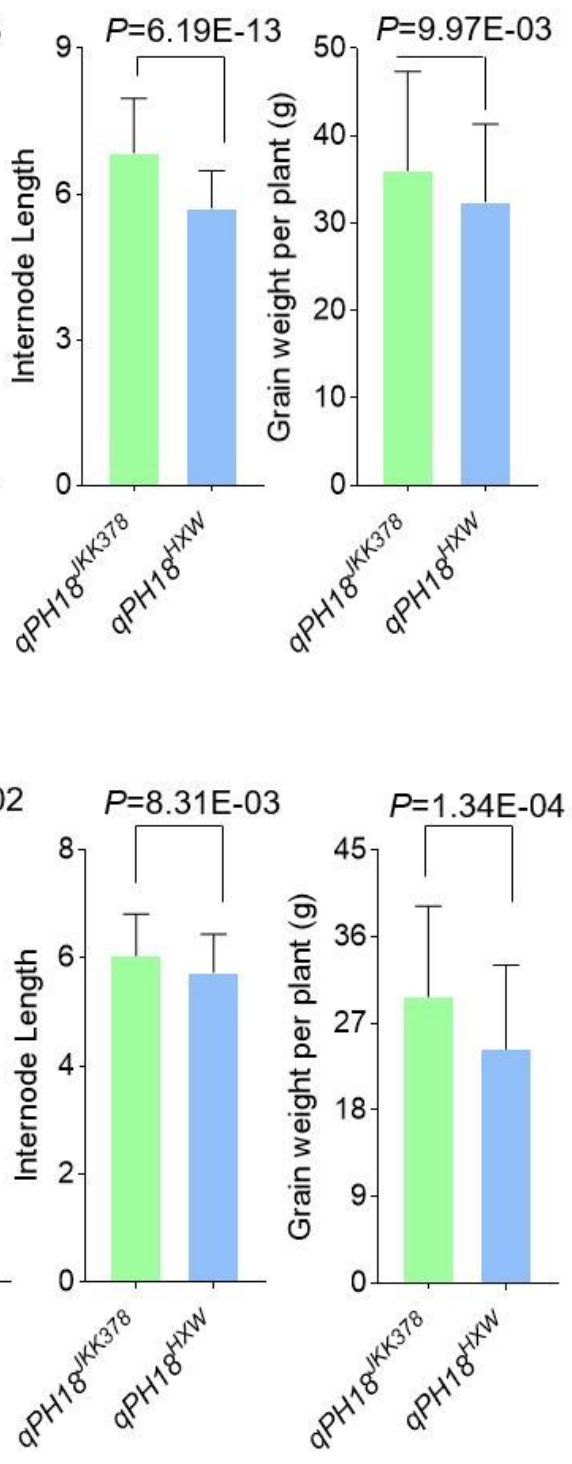

\section{Figure 2}

Phenotypes of plant height, node number, internode length, and grain weight per plant of subgroups containing the allele of qPH18JKK378 and qPH18HXW. a: data of 2018. b: data of 2019. 
a

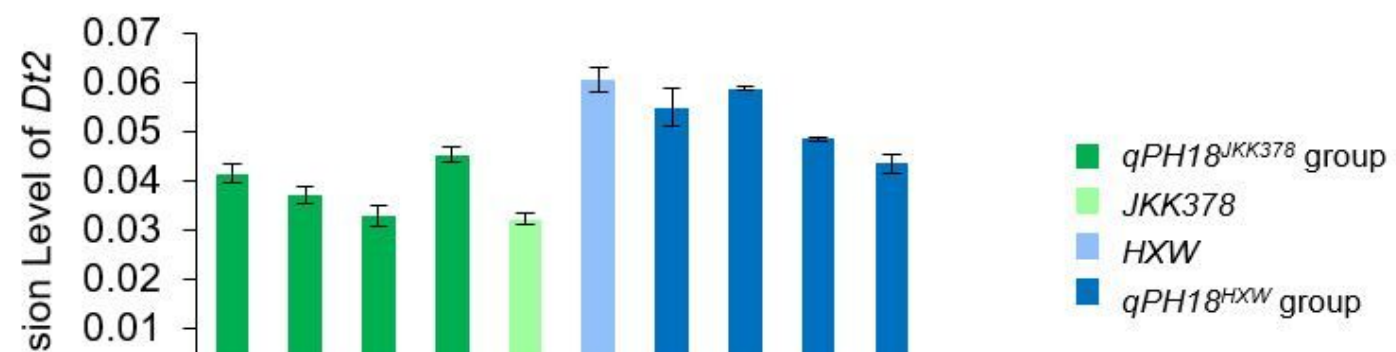

b
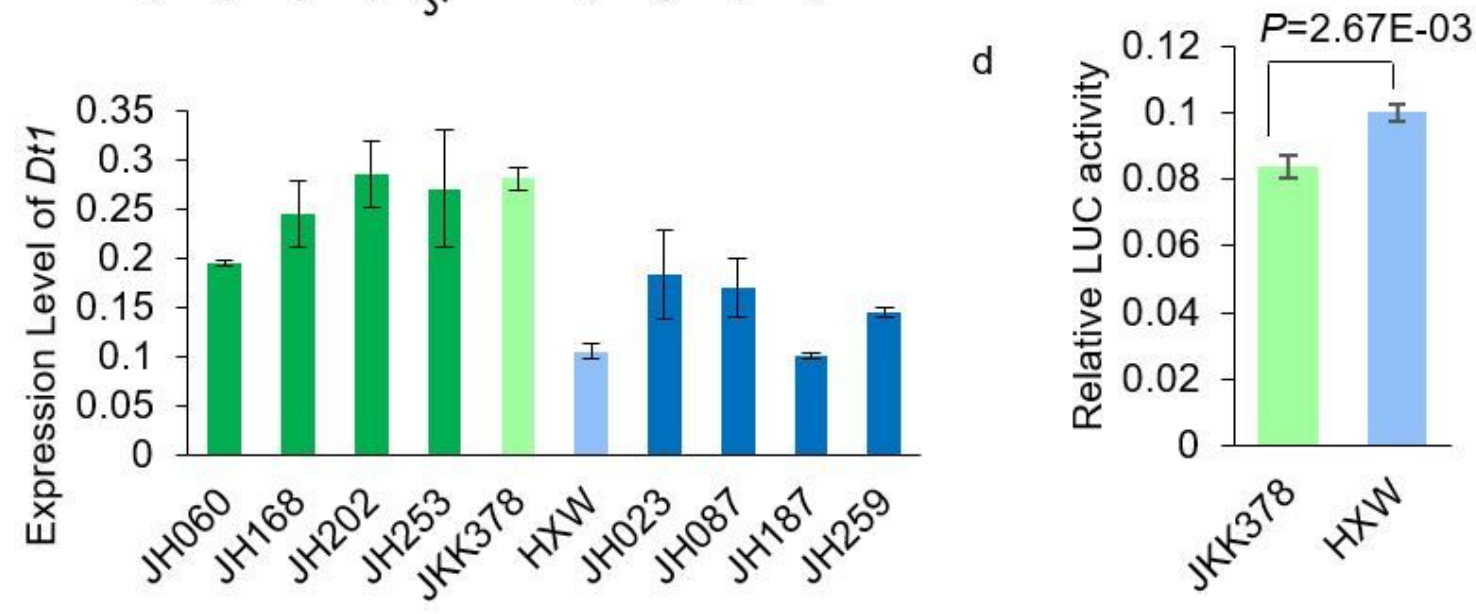

C

\section{\begin{tabular}{|l|l|l|l|}
\hline 35S promoter & REN & JKK378-Dt2 promoter & LUC \\
\hline
\end{tabular}}

\begin{tabular}{l|l|l|l}
3 35S promoter & REN & HXW-Dt2 promoter & LUC \\
\hline
\end{tabular}

Figure 3

qRT-PCR analysis and luciferase activity of Candidate Gene. a: Expression level of Dt2 by qRT-PCR between subgroups containing the allele of qPH18JKK378 and qPH18HXW. b: Expression level of Dt1 by qRT-PCR between subgroups containing the allele of qPH18JKK378 and qPH18HXW. The relative expression levels are normalized to GmTubulin. The data are means \pm SD of three biological replicates. Two-tailed, paired-sample t-tests were used to generate the $P$ value. c: Constructs used for the transient transfection assay in Arabidopsis protoplast cells. d: Luciferase activity regulated under control of Dt2 promoter.

\section{Supplementary Files}

This is a list of supplementary files associated with this preprint. Click to download.

- Sfig1.jpg

- Sfig2.jpg

- Sfig3.jpg

- Sfig4.jpg

- Sfig5.jpg 